\title{
Recurrent invasive Haemophilus influenzae serotype a infection in an infant
}

Kathleen Whyte ${ }^{1 *}$, Paul N. Levett ${ }^{2}$, Greg B. Horsman ${ }^{2}$, Khami Chokani ${ }^{3}$, Kristy Hayden ${ }^{1}$, Michelle Shuel ${ }^{1}$ and Raymond S. W. Tsang ${ }^{1}$

*Correspondence: kathleen.whyte@phac-aspc.gc.ca

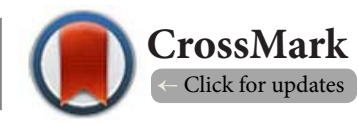

${ }^{1}$ Vaccine Preventable Bacterial Diseases, National Microbiology Laboratory, Winnipeg, MB, Canada.

${ }^{2}$ Saskatchewan Disease Control Laboratory, University of Regina, Regina, SK, Canada.

${ }^{3}$ Prince Albert Parkland Health Region, Prince Albert, SK, Canada.

\begin{abstract}
Background: Before introduction of conjugated Haemophilus influenzae serotype b (Hib) vaccines into the routine childhood immunization programs, Hib was a major cause of meningitis in infants and children under the age of 5 . In the post-Hib vaccine era, the epidemiology of invasive H. influenzae has changed substantially with most invasive diseases now caused by non-Hib strains, including H. influenzae serotype a (Hia) and serotype f, as well as non-encapsulated or non-typeable strains. This case report describes the microbiology of Hia in a recurrent invasive infection in an infant. The Hia strain involved is described together with current knowledge of Hia infection including methods of protection.

Methods: Isolates were characterised by Gram stain, growth factor requirements, biotype, serotype, detection of IS1016-bexA deletion, multilocus sequence typing, pulsed-field gel electrophoresis and antimicrobial susceptibility testing.

Results: All three isolates appeared to be identical, belonged to biotype II, serotype a, sequence type 23, lacked the IS1016-bexA partial deletion, and were susceptible to commonly prescribed antibiotics tested.

Conclusion: Hia has emerged as a significant invasive pathogen in the post Hib vaccine era. MLST and PFGE serve as useful techniques for typing of Hia. Many attributes of Hia and the disease it causes bear resemblance to Hib and Hib disease, including the ability to cause recurrent infections. This raises the potential for protection by vaccination and chemoprophylaxis.
\end{abstract}

Keywords: Haemophilus influenzae, serotype a, recurrent infection, Hib

\section{Introduction}

Haemophilus influenzae is responsible for causing a number of invasive (meningitis, septicemia, septic arthritis, etc.) and non-invasive (otitis media, bronchitis, sinusitis, etc.) infections. $H$. influenzae isolates may or may not produce a polysaccharide capsule and are designated as encapsulated, or non-encapsulated, respectively. Encapsulated strains can be further characterized as belonging to one of serotypes a, b, c, d, $\mathrm{e}$, or $\mathrm{f}$ based on their capsular structure, and non-encapsulated strains are designated as non-typeable.

A conjugate Hib vaccine was developed and introduced into the routine immunization schedule in many countries, including Canada in the early 1990s. Decreased instance of Hib disease has since been observed [1], permitting other serotypes and non-typeable strains to become more prevalent in some regions, as indicated by routine surveillance.

Here we report a case of recurrent invasive infection in an infant due to Hia. We characterized the strains isolated from the infant during the two episodes of infection, and we discussed our current knowledge of Hia infection together with potential methods of protection.

\section{Case presentation}

We report a case of recurrent invasive Haemophilus influenzae serotype a (Hia) infection in a 10-month old native infant in Saskatchewan, Canada. The infant's mother was infected with both the human immunodeficiency virus (HIV) and hepatitis $\mathrm{C}$ virus (HCV), and as such the infant received Azidothymidine (AZT) at birth and during infancy as per national guidelines [2]. In November 2013, the patient was admitted to hospital with 
Whyte et al., Microbiology Discovery 2015,

blood culture confirmed diagnosis of Hia septicemia which was successfully treated with a standard antibiotic regime [3] to which the strain was fully susceptible. The patient was discharged without any significant sequelae from the Hia infection. However, the infant was re-admitted to the hospital in February 2014 with suspected sepsis and meningitis, and both CSF and blood cultures grew Hia. Despite vigorous treatment with antibiotics, the infant succumbed to the second episode of the Hia infection. At both admissions to the hospital, the patient remained HIV and HCV negative by PCR detection of the viruses. We believe this case is of interest because it demonstrates how Hia resembles Hib in the pre-Hib vaccine era, in potentially causing severe recurrent systemic infections. The resemblance of Hia to Hib may raise the possibility of potential control through chemoprophylaxis and ultimately development of a Hia conjugate vaccine.

\section{Investigations}

Identification was based on Gram stain morphology, growth requirement for $\mathrm{X}$ and $\mathrm{V}$ factors, and standard biochemical tests [4]. Biotyping was determined by biochemical reactions for urease, indole and ornithine decarboxylase [5]. Serotype was determined by the slide agglutination method using commercial antisera (Difco, Becton Dickinson, Oakville, Ontario, (Canada), and confirmed by PCR amplification of serotype specific and capsule transport, bexA, genes [6]. Detection of deletion involving parts of the IS1016 and bexA genes in the capsule synthesis operon was done as previously described [7].

For multilocus sequence typing (MLST), 7 housekeeping genes ( $a d k$, atpG, frdB, fucK, $m d h, p g i$, recA) were amplified by PCR and sequenced as previously described [8]. The MLST website (http://haemophilus.mlst.net) was used to assign allele numbers and sequence types. For pulsed-field gel electrophoresis (PFGE), cultures were suspended in 100 $\mathrm{mM}$ Tris-EDTA buffer and adjusted to a turbidity of 0.5 . The suspension was mixed with $1.5 \%$ SeaKem Gold Agarose (Lonza, Cedarlane, Burlington, North Carolina, USA), to form plugs. The plugs were treated with lysis buffer and washed to yield genomic DNA free from any residual reagents. Plug slices were digested with Smal restriction enzyme (Invitrogen, Burlington, Ontario, Canada) and PFGE was performed using the CHEF-DR III unit (Bio-Rad Laboratories, Mississauga, Ontario, Canada). Besides the isolates from the current case, other Hia described in our previous study [9] were included for comparison.

Antibiotic susceptibility disk diffusion testing was conducted according to CLSI guidelines [10], and $\beta$-lactamase production was determined using DrySlide Nitrocefin (Becton Dickinson, Oakville, Ontario, Canada).

\section{Characterization of the Hia isolated from this case}

The blood culture isolate from the first hospitalization and the isolates from the blood and CSF cultures from the second hospitalization three months later were identified as biotype
II H. influenzae. Slide agglutination with serotyping antisera revealed all three isolates as serotype a, and they were confirmed to contain the bexA and the serotype a-specific genes by PCR. MLST revealed all three isolates belonging to ST23. They lacked the IS1016-bexA partial deletion in their capsule synthesis (cps) operon. All three isolates did not produce $\beta$-lactamases, and were susceptible to ampicillin, amoxicillinclavulanic acid, cefaclor, ceftriaxone, chloramphenicol, tetracycline, azithromycin, clarithromycin, ciprofloxacin, moxifloxacin, levofloxacin, imipenem, meropenem, and trimethoprim-sulfamethoxazole. PFGE also showed identical DNA fingerprints for the three Hia isolates (Figure 1).

\section{Discussion}

The capsule of Hia is most similar to Hib but very different from capsules of the other serotypes. Hia capsule is made up of a polymer of a di-saccharide of glucose-ribitol phosphate [11] while the polymer of Hib capsule is ribose-ribitol phosphate [12]. Experimental studies in animals using isogenic mutants that differ from each other by their capsule structures reveal that Hib is the most virulent serotype followed by Hia, and then other serotypes [13].

In this case of a recurrent Hia systemic infection, although the bacteria isolated from the patient at the hospital during both episodes of infection were identical, the clinical findings suggested that it was likely a re-infection rather than relapse. First the isolates involved were highly sensitive to the antibiotics tested as well as to the antibiotic used to

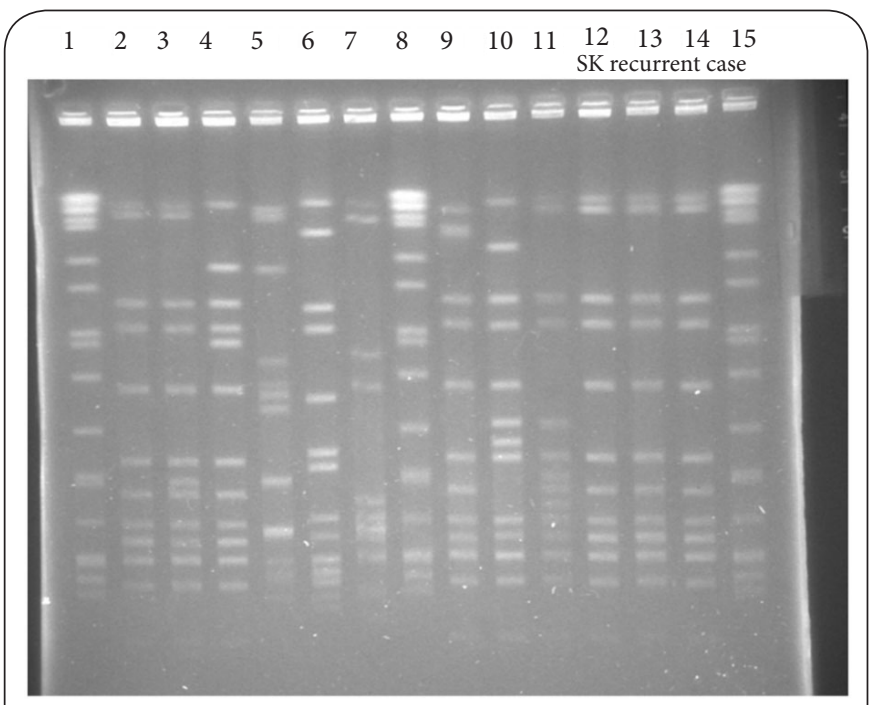

Figure 1. Pulsed-field gel electrophoresis of Haemophilus influenzae strains, comparing DNA fingerprints from isolates obtained from the recurrent case in Saskatchewan (SK) with $H$. influenzae strains representing different Sequence Type (ST). Lanes 1, 8 and 15 were profiles from Salmonella braenderup as markers; Lanes 12, 13 and 14 were profiles from $H$. influenzae serotype a isolates of the recurrent case. Lanes 2, 3, 4 and 11, ST-23; Lane 5, ST-372; Lane 6, ST-4; Lane 7 ST-62; Lane 9, ST-56, Lane 10, ST-529. 
treat the initial episode of the infection. The patient fully recovered, and was discharged without any noted abnormality. Secondly, the patient appeared well during the three month period between the infections. The fact that all three strains recovered from the patient in the two episodes of infection were identical merely reflected the common nature of this clone of Hia in Canada [9].

Indeed, of the 116 Hia isolates collected from 1995-2012 at the NML from Canadian sources, 95 (82\%) belonged to ST-23, and another 19 (16\%) belonged to STs related to ST23 or being part of the ST-23 clonal complex [9]. The lack of $\beta$-lactamase in the $3 \mathrm{Hia}$ isolates in this case as well as their uniform sensitivity to the commonly prescribed antibiotics are also common features of Hia in Canada [14], which are in stark contrast to Hib which are more commonly found to have either $\beta$-lactamases and/or resistance to antibiotics. Despite susceptibility to antibiotics and aggressive antibiotic treatment, the infant succumbed to the re-infection which may highlight the significance of invasive Hia infections, similar to invasive Hib disease before the introduction of the Hib conjugate vaccine [15]. Another feature in this case noteworthy to mention is the ethnicity of the patient who was described as aboriginal. Related to this point are (a) Hia has recently emerged as a significant invasive pathogen in the aboriginal population in North America in the post-Hib vaccine era [16-18]; (b) in the pre-Hib vaccine era, aboriginal communities in North America have reportedly the highest incidence rates of invasive Hib disease in the world [19-21]; (c) recurrent infections due to Hib [22] and Hia [23] have been reported in the literature. Currently, there are no oropharyngeal carriage studies of Hia in aboriginal and nonaboriginal communities in Canada and North America to understand the prevalence of this organism circulating in the population, which probably serves as a source of infection, including the recurrent infection described herein.

Another contributing factor to recurrent $\mathrm{Hib}$ (and possibly Hia) infection in young children is the poor immunogenicity of the plain Hib (and likely Hia) capsular polysaccharide and the immature nature of the immune system in young children who do not respond to plain polysaccharide vaccines. The poor immunogenicity of plain polysaccharide vaccines in young children can be overcome by conjugation of the polysaccharide to a carrier protein such as tetanus toxoid. Additional contributing factors may involve potential genetic polymorphism in the antibody encoding genes or genetic loci that may affect antibody acquisition and $\mathrm{Hib}$ (possibly $\mathrm{Hia}$ ) disease susceptibility $[\mathbf{2 4}, \mathbf{2 5}]$. However, these claims have not been substantiated by further systematic studies.

Besides meningitis [26], Hia has been reported to cause sepsis with toxic shock [27], septic arthritis [28], soft tissue infection with pus and abscess [29], pneumonia with empyema [30], and epiglottitis [31]. Most invasive Hia cases occur in children between the ages of 6 months to 2 years [32; authors' unpublished data]. Case fatality rates have been reported from as low as 2 to $5 \%$ [32-34] to as high as 16 to $23 \%[35,36]$ and even $33 \%$ with infection due to strains possessing the IS1016-bexA deletion [37]. This vast range of case fatality rates may be related to the patient population as well as to the strains of Hia involved. Previous studies have shown that strains that belong to ST-4 and contain the IS1016-bexA partial deletion are associated with higher case fatality rates $[37,38]$. However, in this case, the isolates involved did not possess this genetic deletion and belonged to ST-23. Nevertheless, the infant succumbed to the infection, which may suggest that this common clone of Hia in Canada is still virulent and has the potential to cause fatal infection, similar to the clinical diseases caused by Hib. The spectrum of invasive diseases, their mortality rates as well as ages of the affected subjects are all very similar to the picture associated with $\mathrm{Hib}$ in the pre-Hib vaccine era.

\section{Conclusions}

The similarities in the microbiology of Hia and Hib as well as the diseases caused by them may suggest that prevention strategies employed for control of invasive Hib disease may be applicable for control of Hia. For example, it is known that close contacts of those with invasive Hib infection are at an increased risk of contracting Hib infection, when compared to the general public, and chemoprophylaxis is recommended for prevention of secondary Hib cases $[3,39]$. Whether or not chemoprophylaxis offered to household as well as other close contacts of this case would prevent the reinfection in this child cannot be known for sure but should be considered for future studies. The capsule polysaccharide of $\mathrm{Hib}$ is a known protective antigen and conjugate vaccine prepared from this antigen has been successfully used to control invasive Hib disease. Conjugated vaccine prepared with the Hia capsular polysaccharide has been proposed as a potential vaccine candidate for prevention of Hia infection [40]. A public health driven vaccine initiative has been established within the Canadian federal government to examine the potential of developing a conjugated Hia vaccine for protection against invasive Hia disease in the Aboriginal population $[18,41]$.

\section{Competing interests}

The authors declare that they have no competing interests.

Authors' contributions
\begin{tabular}{|l|c|c|c|c|c|c|c|}
\hline Authors' contributions & KW & PNL & GBH & KC & KH & MS & RSWT \\
\hline Research concept and design & -- & $\checkmark$ & $\checkmark$ & $\checkmark$ & -- & -- & $\checkmark$ \\
\hline Collection and/or assembly of data & $\checkmark$ & $\checkmark$ & -- & $\checkmark$ & $\checkmark$ & $\checkmark$ & -- \\
\hline Data analysis and interpretation & $\checkmark$ & $\checkmark$ & -- & $\checkmark$ & -- & $\checkmark$ & $\checkmark$ \\
\hline Writing the article & $\checkmark$ & $\checkmark$ & -- & $\checkmark$ & -- & -- & $\checkmark$ \\
\hline Critical revision of the article & $\checkmark$ & $\checkmark$ & $\checkmark$ & $\checkmark$ & -- & -- & $\checkmark$ \\
\hline Final approval of article & $\checkmark$ & $\checkmark$ & $\checkmark$ & $\checkmark$ & $\checkmark$ & $\checkmark$ & $\checkmark$ \\
\hline Statistical analysis & -- & -- & -- & -- & -- & -- & -- \\
\hline
\end{tabular}

Acknowledgement

The authors would like to thank Dennis Law for carrying out 
Whyte et al., Microbiology Discovery 2015,

serotype confirmation by PCR; and the staff at the National Microbiology Laboratory DNA Core Facility for performing the sequencing work. This publication made use of data from the Multi Locus Sequence Typing website (http://haemophilus.mlst. net/) at Imperial College London, developed by David Aanensen and funded by Welcome Trust.

Publication history

Editor: António Pedro Fonseca, University of Porto, Portugal. EIC: Todd R. Callaway, Food and Feed Safety Research Unit, USA. Received: 08-Jun-2015 Final Revised: 08-Jul-2015

Accepted: 10-Jul-2015 Published: 17-Jul-2015

\section{References}

1. Hviid $A$ and Melbye M. Impact of routine vaccination with a conjugate Haemophilus influenzae type b vaccine. Vaccine. 2004; 22:378-82. | Article | PubMed

2. Care of the infant born to an HIV-positive mother. Paediatr Child Health. 2000; 5:161-70. | PubMed Abstract | PubMed Full Text

3. American Academy of Pediatrics: Summary of infectious diseases, Section 3, Haemophilus influenzae infections. In: Pickering LK, Baker CJ, Kimberlin DW, Long SS (Eds.), Red Book: 2010 Report of the Committee on Infectious Diseases. 2012; 345-352.

4. Ledeboer NA and Doern GV: Haemophilus. Versalovic J, Carroll KC, Funke G, Jorgensen JH, Landry ML and Warnock DW (Eds.), Manual of Clinical Microbiology. $10^{\text {th }}$ edition. 2011; 1:588-602.

5. Kilian M. A taxonomic study of the genus Haemophilus, with the proposal of a new species. J Gen Microbiol. 1976; 93:9-62. | Website | PubMed

6. Falla TJ, Crook DW, Brophy LN, Maskell D, Kroll JS and Moxon ER. PCR for capsular typing of Haemophilus influenzae. J Clin Microbiol. 1994; 32:2382-6. | Article | PubMed Abstract | PubMed Full Text

7. Kroll JS, Moxon ER and Loynds BM. Natural genetic transfer of a putative virulence-enhancing mutation to Haemophilus influenzae type a. J Infect Dis. 1994; 169:676-9. I Article | PubMed

8. Meats E, Feil EJ, Stringer S, Cody AJ, Goldstein R, Kroll JS, Popovic T and Spratt BG. Characterization of encapsulated and noncapsulated Haemophilus influenzae and determination of phylogenetic relationships by multilocus sequence typing. J Clin Microbiol. 2003; 41:1623-36. | Article | PubMed Abstract | PubMed Full Text

9. Tsang RS, Shuel M, Wylie J, Lefebvre B, Hoang L and Law DK. Population genetics of Haemophilus influenzae serotype a in three Canadian provinces. Can J Microbiol. 2013; 59:362-4. | Article I PubMed

10. Clinical and Laboratory Standards Institute: Performance Standards for Antimicrobial Susceptibility Testing. Clinical and Laboratory Standards Institute. Eighteenth Informational Supplement. CLSI Document M100-S23. 2013.

11. Branefors-Helander P. The structure of the capsular antigen from Haemophilus influenzae type A. Carbohydr Res. 1977; 56:117-22. | Article I PubMed

12. Crisel RM, Baker RS and Dorman DE. Capsular polymer of Haemophilus influenzae, type b. I. Structural characterization of the capsular polymer of strain Eagan. J Biol Chem. 1975; 250:4926-30. | Article I PubMed

13. Zwahlen A, Kroll JS, Rubin LG and Moxon ER. The molecular basis of pathogenicity in Haemophilus influenzae: comparative virulence of genetically-related capsular transformants and correlation with changes at the capsulation locus cap. Microb Pathog. 1989; 7:225-35. | Article | PubMed

14. Shuel M, Whyte K, Drew T, Wylie J, Lefebvre B, Hoang L and Tsang RS. Differential susceptibility of invasive Haemophilus influenzae serotype a and serotype $\mathbf{b}$ to ampicillin and other commonly prescribed antibiotics. Lett App/ Microbiol. 2014; 59:193-9. | Article | PubMed

15. Adderson EE, Byington CL, Spencer L, Kimball A, Hindiyeh M, Carroll K, Mottice S, Korgenski EK, Christenson JC and Pavia AT. Invasive serotype a Haemophilus influenzae infections with a virulence genotype resembling Haemophilus influenzae type b: emerging pathogen in the vaccine era? Pediatrics. 2001; 108:E18. | Article I PubMed

16. Ulanova M, Tsang R and Altman E. Neglected infectious diseases in Aboriginal communities: Haemophilus influenzae serotype a and Helicobacter pylori. Vaccine. 2012; 30:6960-6. | Article | PubMed

17. Bruce MG, Zulz T, DeByle C, Singleton R, Hurlburt D, Bruden D, Rudolph K, Hennessy T, Klejka J and Wenger JD. Haemophilus influenzae serotype a invasive disease, Alaska, USA, 1983-2011. Emerg Infect Dis. 2013; 19:932-7. | Article | PubMed Abstract | PubMed Full Text

18. Tsang RS, Bruce MG, Lem M, Barreto $L$ and Ulanova $M$. A review of invasive Haemophilus influenzae disease in the Indigenous populations of North America. Epidemiol Infect. 2014; 142:1344-54. | Article | PubMed

19. Losonsky GA, Santosham M, Sehgal VM, Zwahlen A and Moxon ER. Haemophilus influenzae disease in the White Mountain Apaches: molecular epidemiology of a high risk population. Pediatr Infect Dis. 1984; 3:539-47. | PubMed

20. Ward JI, Lum MK, Hall DB, Silimperi DR and Bender TR. Invasive Haemophilus influenzae type b disease in Alaska: background epidemiology for a vaccine efficacy trial. J Infect Dis. 1986; 153:17-26. | Article | PubMed

21. Hammond GW, Rutherford BE, Malazdrewicz R, MacFarlane N, Pillay N, Tate RB, Nicolle LE, Postl BD and Stiver HG. Haemophilus influenzae meningitis in Manitoba and the Keewatin District, NWT: potential for mass vaccination. CMAJ. 1988; 139:743-7. | PubMed Abstract | PubMed Full Text

22. Brenneman G, Silimperi D and Ward J. Recurrent invasive Haemophilus influenzae type b disease in Alaskan Natives. Pediatr Infect Dis J. 1987; 6:388-92. | PubMed

23. Hammitt LL, Block S, Hennessy TW, Debyle C, Peters H, Parkinson A, Singleton R and Butler JC. Outbreak of invasive Haemophilus influenzae serotype a disease. Pediatr Infect Dis J. 2005; 24:453-6. | Article | PubMed

24. Feeney AJ, Atkinson MJ, Cowan MJ, Escuro G and Lugo G. A defective Vkappa A2 allele in Navajos which may play a role in increased susceptibility to haemophilus influenzae type b disease. J Clin Invest. 1996; 97:2277-82. | Article | PubMed Abstract | PubMed Full Text

25. Petersen GM, Silimperi DR, Rotter JI, Terasaki PI, Schanfield MS, Park MS and Ward JI. Genetic factors in Haemophilus influenzae type b disease susceptibility and antibody acquisition. J Pediatr. 1987; 110:228-33. | Article | PubMed

26. de Padua RA, de Lima Scodro RB, Ghiraldi LD, Siqueira VL, Yamashita YK, Helbel $C$ and Cardoso RF. Haemophilus influenzae serotype a meningitis. Ann Clin Lab Sci. 2009; 39:405-8. | Article | PubMed

27. Francis J, Anders M, Lobegeier P and Nourse C. Fatal Haemophilus influenzae type a sepsis in an infant. J Paediatr Child Health. 2013; 49:E235-8. | Article | PubMed

28. Fischer NJ. Haemophilus influenzae serotype a septic arthritis in an immunized central Australian indigenous child. Int J Infect Dis. 2014; 21:15-6. | Article | PubMed

29. Bezuhly M and Fish JS. Haemophilus influenzae serotype a as the causative agent of a pediatric upper extremity infection. Hand (NY). 2012; 7:94-7. | Article | PubMed Abstract | PubMed Full Text

30. Rutherford GW and Wilfert CM. Invasive Haemophilus influenzae type a infections: a report of two cases and a review of the literature. Pediatr Infect Dis. 1984; 3:575-7. I PubMed

31. Cerqueira AM, Tsang RSW, Jamieson FB and Ulanova M. A case of acute epiglottitis caused by Haemophilus influenzae type a in an adult. JMM Case Reports. 2014. | Article

32. Rotondo JL, Sherrard L, Helferty M, Tsang R and Desai S. The epidemiology of invasive disease due to Haemophilus influenzae serotype a in the Canadian North from 2000 to 2010. Int J Circumpolar Health. 2013; 72:1-5. | Article I PubMed Abstract | PubMed Full Text

33. Millar EV, O'Brien KL, Watt JP, Lingappa J, Pallipamu R, Rosenstein N, Hu $D$, Reid R and Santosham M. Epidemiology of invasive Haemophilus 
Whyte et al., Microbiology Discovery 2015,

http://www.hoajonline.com/journals/pdf/2052-5958-3-4.pdf

influenzae type A disease among Navajo and White Mountain Apache children, 1988-2003. Clin Infect Dis. 2005; 40:823-30. | Article | PubMed

34. Bruce MG, Deeks SL, Zulz T, Navarro C, Palacios C, Case C, Hemsley C, Hennessy T, Corriveau A, Larke B, Sobel I, Lovgren M, Debyle C, Tsang R and Parkinson AJ. Epidemiology of Haemophilus influenzae serotype a, North American Arctic, 2000-2005. Emerg Infect Dis. 2008; 14:48-55. | Article | PubMed Abstract | PubMed Full Text

35. Ribeiro GS, Reis JN, Cordeiro SM, Lima JB, Gouveia EL, Petersen M, Salgado K, Silva HR, Zanella RC, Almeida SC, Brandileone MC, Reis MG and Ko Al. Prevention of Haemophilus influenzae type b (Hib) meningitis and emergence of serotype replacement with type a strains after introduction of Hib immunization in Brazil. J Infect Dis. 2003; 187:109-16. | Article | PubMed

36. McConnell A, Tan B, Scheifele D, Halperin S, Vaudry W, Law B and Embree $\mathrm{J}$. Invasive infections caused by haemophilus influenzae serotypes in twelve Canadian IMPACT centers, 1996-2001. Pediatr Infect Dis J. 2007; 26:1025-31. | Article | PubMed

37. Lima JB, Ribeiro GS, Cordeiro SM, Gouveia EL, Salgado K, Spratt BG, Godoy D, Reis MG, Ko Al and Reis JN. Poor clinical outcome for meningitis caused by Haemophilus influenzae serotype $A$ strains containing the IS1016-bexA deletion. J Infect Dis. 2010; 202:1577-84. | Article | PubMed Abstract | PubMed Full Text

38. Kapogiannis BG, Satola S, Keyserling HL and Farley MM. Invasive infections with Haemophilus influenzae serotype a containing an IS1016-bexA partial deletion: possible association with virulence. Clin Infect Dis. 2005; 41:e97-103. | Article | PubMed

39. Gkentzi D, Collins S, Ramsay ME, Slack MP and Ladhani S. Revised recommendations for the prevention of secondary Haemophilus influenzae type b (Hib) disease. J Infect. 2013; 67:486-9. | Article | PubMed

40. Jin Z, Romero-Steiner S, Carlone GM, Robbins JB and Schneerson R. Haemophilus influenzae type a infection and its prevention. Infect Immun. 2007; 75:2650-4. | Article | PubMed Abstract | PubMed Full Text

41. Desai S, Tsang R, St-Laurent $M$ and Cox A. Collaboration on a public health-driven vaccine initiative. CCDR. 2014; 40:365-368. | Article

\section{Citation:}

Whyte K, Levett PN, Horsman GB, Chokani K, Hayden $\mathrm{K}$, Shuel M and Tsang RSW. Recurrent invasive Haemophilus influenzae serotype a infection in an infant. Microbiol Discov. 2015; 3:4.

http://dx.doi.org/10.7243/2052-6180-3-4 